\title{
SOIL-PILE-STRUCTURE INTERACTION EFFECTS ON HIGH-RISE BUILDING UNDER SEISMIC SHAKING
}

\author{
Umesh Jung Thapa ${ }^{1 *}$, Ramesh Karki ${ }^{2}$
}

\begin{abstract}
In this paper, study of the response (base shear, time period, storey drift, storey displacement) of a structure is done for the tall building including basement with fixed base and with pile foundation considering Soil Structure Interaction (SSI). Finite element based program ETABS2016 v16.1.0 is used for the analysis of the superstructure. Seismic analysis is done to get the dynamic response of superstructure for two types of model,one model is with fixed baseand second is Model with Winkler spring for Chhaya Center, Thamel, a high rise building with 14 story including double basements. Itisobserved with the consideration of Soil Structure Interaction (SSI). The soil is replaced by spring and assigned at joints. EI Centro earthquake (1940) is used for time history analysis. The response obtained due to SSI effect is compared with fixed based model. Results of analysis presented include the comparison of natural periods, base shears, displacements and overturning moment. It is observed that the natural periods increase and the base shears decrease as the base become more flexible.
\end{abstract}

\section{INTRODUCTION}

Structures founded on rock are considered to be fixed base structure. Computation of their response is relatively simple. On the other hand, the same structure would respond differently if supported on soft deposit. First, the inability of the foundation to confirm to the deformations of the free field motion. Second, the dynamic response of the structure itself would induce deformation of the supporting soil. This process, in which the response of the soil influences the motion of the structure and the response of the structure influence the motion of the soil, is referred to as soil structure interaction.

\subsection{Types of Analysis}

\subsubsection{Direct Analysis}

Direct Analysis method is the one in which the soil and substructure are modeled together in a single step accounting for both inertial and kinematic interaction. Inertial interaction develops in structure due to own vibrations give rise to base shear and base moment, which in turn cause displacements of the foundation relative to free field. Kinematic interaction develops due to presence of stiff foundation elements on or in soil cause foundation motion to deviate from free field motions.

\subsubsection{Substructure Approach}

Sub-Structure Method is one in which the analysis is broken down into several steps that

*1_Postgraduate student, IoE, Email:umeshjthapa@gmail.com

*2Assistant professor, Thapathali Campus, Email:rameshkarki@ioe.edu.np 
is the principal of superposition is used to isolate the two primary causes of soilstructure interaction, inability of foundation to match the free field deformation and the effect of dynamic response of structure foundation system on the movement of supporting soil.

\subsection{Problem Statement}

Conventional structural design methods neglect the SSI effects. Neglecting SSI is reasonable for light structures in relatively stiff soil such as low rise buildings and sample rigid retaining walls. The effect of SSI, however, becomes prominent for heavy structures resting on relatively soft soils for example nuclear power plants, high-rise buildings and elevated-highways on soft soil.

A controversial issue in the seismic analysis and design of high rise building lies in incorporating the effects of the seismic response of these structures. Building codes lack recommendations concerning this controversy; thus, the designers are basing their analysis on approximations, engineering judgment and experience. This has been an active area of research throughout the past decade: (Dutta and Roy, 2002), (Dutta et al., 2004), (Shakib, 2004), (Naim et al., 2008), (El Ganainy and El Naggar, 2009), (Raychowdury 2010), (Tabatabaeifar and Massumi, 2010). The soil-structure interaction (SSI) is a complicated phenomenon for structures coupled with the soil medium, which is generally semi-infinite in extent and nonlinear in its material behavior. The problem of SSI in the seismic analysis of highrise buildings with pile foundation have become increasingly important, as it may be inevitable to build such a structures for the sites with less favorable geotechnical conditions due to ever-increasing difficulty in acquiring new construction sites.

Damage sustained in recent earthquakes, such as the 2015 Gorkha earthquake, 1995 Kobe earthquake, have also highlighted that the seismic behavior of a structure is highly influenced not only by the response of the superstructure, but also by the response of the foundation and the ground as well.

\section{LITERATURE REVIEW}

Few research has been conducted related to seismic response of high rise building with pile foundation due to soil structure interaction.

Dr. SushmaPulikanthi and Prof. Pradeep Kumar Ramancharla,2013 observed that there is two times increase in the acceleration response of the top floor while considering the SFSI over fixed base analysis for nonlinear case of buildings supported on pile foundation under transient loading.

Kraus \& D.dzakic,2013 observed that story drift is increased when the soil is modeled using Winkler springs.

MuberraEserAydemir investigated the seismic behavior of multi storied structures considering SSI according to Turkish Seismic design code and he found that there is 
increase in natural period of the structure considering SSI corresponding to rigidly supported structure.

\section{METHODOLOGY}

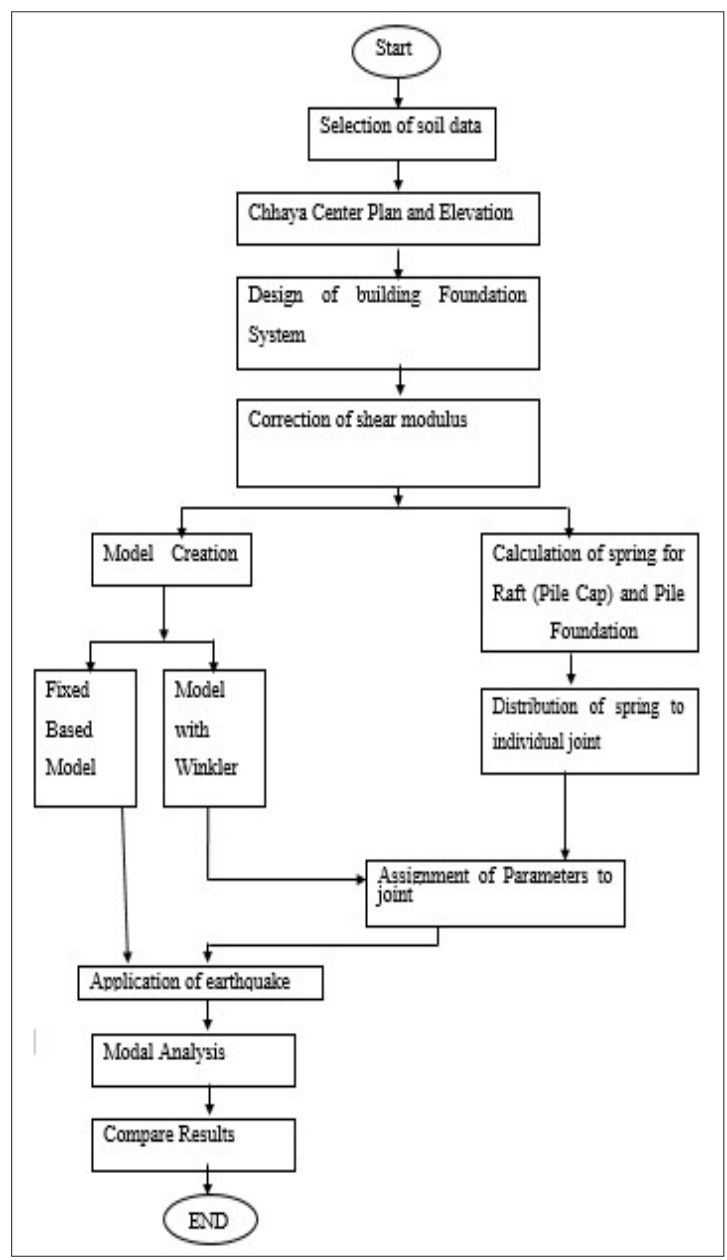

3.1 Method Adopted

- $\quad$ Simplified Approach

- $\quad$ Follows Code ASCE 41-

06

- Follows Hien Manh

Nghiem, "Soil Pile Structure Interaction Effects on high rises under seismic shaking" University of Colorado at Denver and health science, Denver, USA

- The Fixed Support is replaced by one parameter property i.e. Linear Springs

\subsection{Steps of SSI Followed}

1. Calculation of shear modulus

2. Calculation of spring stiffness

3. Distribution of spring on each joint

Fig: Flowchart for the methodology

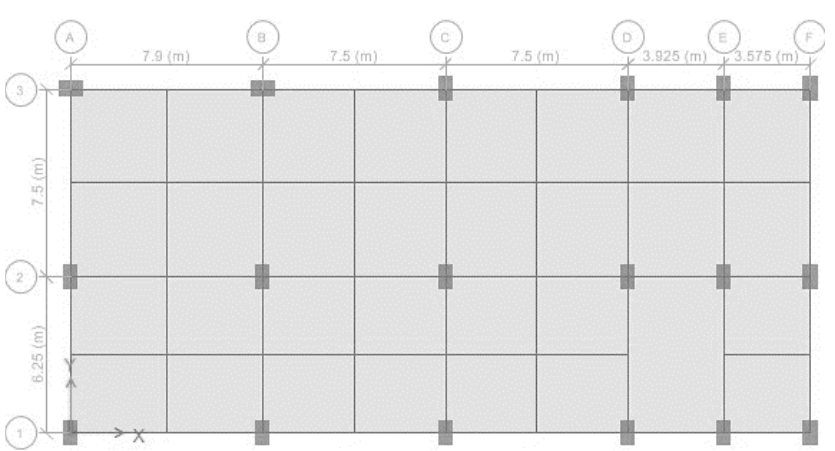

Figure: Typical Plan of Chhaya Center 


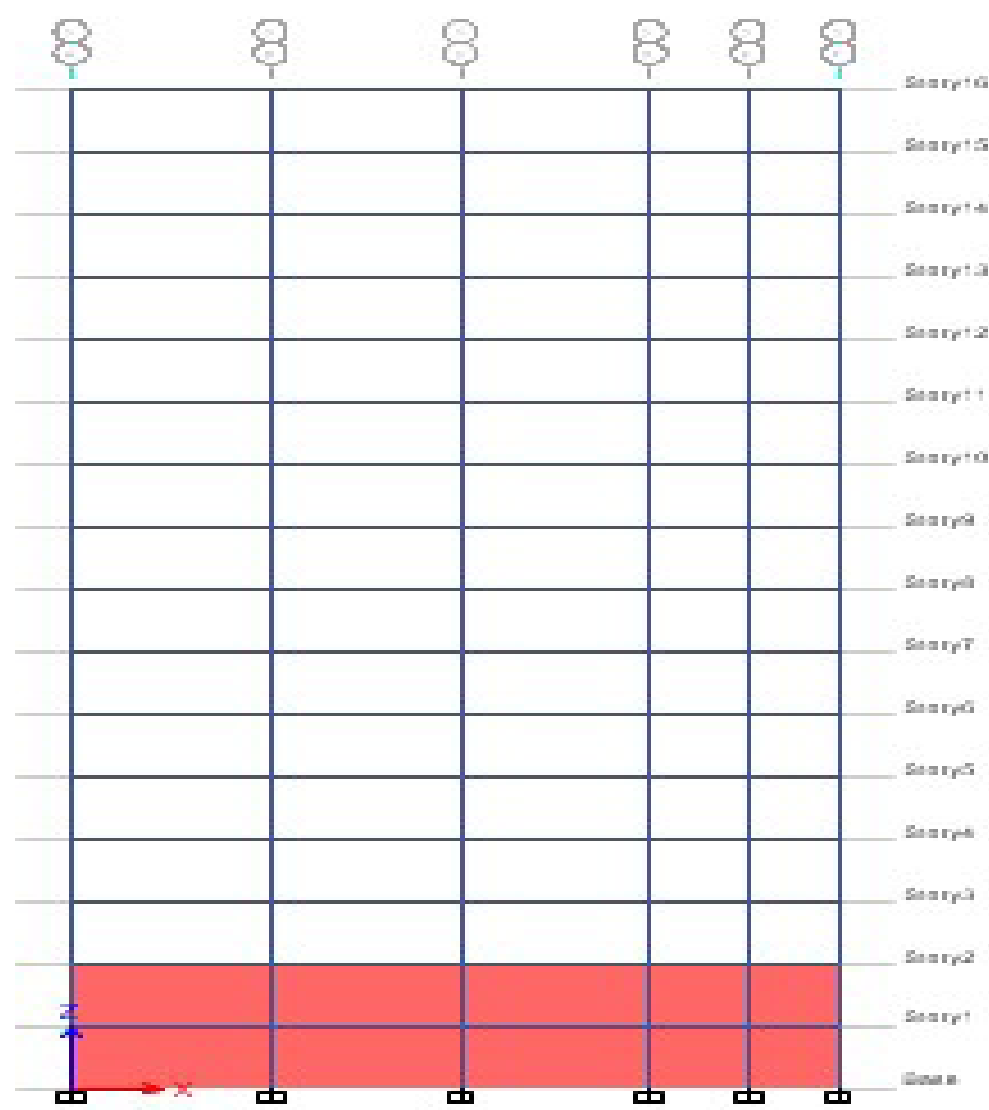

Figure: Typical Elevation of Chhaya Center (16 Story)

3.3 Stiffness for Raft Foundation (Pile Cap), Shear wall and Pile Foundation Table: Formula for Calculating Spring Stiffness (ASCE, 2010)

\begin{tabular}{|l|l}
\hline Degree of Freedom & Stiffness of Foundation at surface \\
\hline Translation along x-axis & $\mathrm{K}_{\mathrm{x}}, \mathrm{sur}=\frac{\mathrm{GB}}{2-\mathrm{v}}\left[3.4\left(\frac{L}{B}\right)^{0.65}+1.2\right]$ \\
\hline Translation along y-axis & $\mathrm{K}_{\mathrm{y}}, \mathrm{sur}=\frac{\mathrm{GB}}{2-\mathrm{v}}\left[3.4\left(\frac{L}{B}\right)^{0.65}+0.4 \frac{L}{B}+0.8\right]$ \\
\hline Translation along z-axis & $\mathrm{K}_{\mathrm{z}}, \mathrm{sur}=\frac{\mathrm{GB}}{1-\mathrm{v}}\left[1.55\left(\frac{L}{B}\right)^{0.75}+0.8\right]$ \\
\hline Rocking about x-axis & $\mathrm{K}_{\mathrm{xx}}, \mathrm{sur}=\frac{\mathrm{GB}^{3}}{1-\mathrm{v}}\left[0.4\left(\frac{\mathrm{L}}{\mathrm{B}}\right)+0.1\right]$ \\
\hline Rocking about y-axis & $\mathrm{K}_{\mathrm{yy}}, \mathrm{sur}=\frac{\mathrm{GB}^{3}}{1-\mathrm{v}}\left[0.47\left(\frac{L}{B}\right)^{2.4}+0.034\right]$ \\
\hline Rocking about z-axis & $\mathrm{K}_{\mathrm{zz}}, \mathrm{sur}=\mathrm{GB}^{3}\left[0.53\left(\frac{L}{B}\right)^{2.45}+0.51\right]$ \\
\hline
\end{tabular}


Table: Formula for Calculating Spring Stiffness (ASCE, 2010)

\begin{tabular}{|l|l|}
\hline Degree of Freedom & Correction Factor for Embedment \\
\hline Translation along x-axis & $\beta=\left(1+0.21 \sqrt{\frac{D}{B}}\right)\left[1+1.6\left(\frac{h d(B+L)}{B L^{2}}\right)^{0.4}\right]$ \\
\hline Translation along y-axis & $\beta_{\mathrm{y}}=\left(1+0.21 \sqrt{\frac{D}{L}}\right)\left[1+1.6\left(\frac{h d(B+L)}{B L}\right)^{0.4}\right]$ \\
\hline Translation along z-axis & $\beta_{\mathrm{z}}=\left(1+\frac{1}{21} \frac{D}{B}\left(2+2.6 \frac{B}{L}\right)\right)\left[1+0.32\left(\frac{d(B+L)}{B L}\right)^{\frac{2}{3}}\right]$ \\
\hline Rocking about x-axis & $\beta_{\mathrm{xx}}=1+2.5 \frac{d}{B}\left[1+\frac{2 d}{B}\left(\frac{d}{D}\right)^{-0.2} \sqrt{\frac{B}{L}}\right]$ \\
\hline Rocking about y-axis & $\beta_{\mathrm{yy}}=1+1.4\left(\frac{d}{L}\right)^{0.6}\left[1.5+3.7\left(\frac{d}{D}\right)^{1.9}\left(\frac{d}{D}\right)^{-0.6]}\right.$ \\
\hline Rocking about z-axis & $\beta_{\mathrm{zz}}=1+2.6\left(1+\frac{\mathrm{B}}{\mathrm{L}}\right)\left(\frac{d}{B}\right)^{0.9}$ \\
\hline
\end{tabular}

\subsection{Stiffness for Pile Foundation}

Hien Manh Nghiem, "Soil Pile Structure Interaction Effects on high rises under seismic shaking" University of Colorado at Denver and health science, Denver, USA

\subsubsection{Torsional Stiffness}

For constant $\mathrm{K}_{\mathrm{qz}}$ or $\mathrm{G}_{\mathrm{s}}$ :

$\mathrm{K}_{66}^{\mathrm{i}}=\frac{\mathrm{L}_{\mathrm{e}}\left(12 \mathrm{G}_{\mathrm{p}} \mathrm{J}_{\mathrm{p}} \mathrm{k}_{\theta z}+\mathrm{k}_{\theta}^{2} \mathrm{~L}_{\mathrm{e}}^{2}\right.}{4\left(3 \mathrm{G}_{\mathrm{p}} \mathrm{J}_{\mathrm{p}}+\mathrm{k}_{\theta \mathrm{z}} \mathrm{L}_{\mathrm{e}}^{2}+3 \mathrm{k}_{\theta z} \mathrm{~L}_{\mathrm{e}}\right)}+\frac{\mathrm{K}_{\theta z \mathrm{~b}}\left(3 \mathrm{G}_{\mathrm{p}} \mathrm{J}_{\mathrm{p}}+\mathrm{k}_{\theta z} \mathrm{~L}_{\mathrm{e}}^{2}\right.}{\left(3 \mathrm{G}_{\mathrm{p}} \mathrm{J}_{\mathrm{p}}+\mathrm{k}_{\theta z} \mathrm{~L}_{\mathrm{e}}^{2}+3 \mathrm{k}_{\theta z \mathrm{~b}} \mathrm{~L}_{\mathrm{e}}\right)} \quad$ (3-1) Where,

$\mathrm{L}_{\mathrm{e}}$ is the Length of the pile.

$\mathrm{G}_{\mathrm{p}}$ is Pile material shear modulus.

$\mathrm{J}_{\mathrm{p}}$ is polar moment of inertia of pile section.

$=4 \pi^{2} \mathrm{G}$

$\mathrm{k}_{\theta b}=\mathrm{r}_{\mathrm{o}}^{3} \mathrm{G}$

\subsubsection{Vertical Stiffness}

For constant $\mathrm{K}_{\mathrm{uz}}$ or $\mathrm{E}_{\mathrm{s}}$ :

$\mathrm{K}_{33}^{\mathrm{i}}=\frac{\mathrm{L}_{\mathrm{e}}\left(12 \mathrm{E}_{\mathrm{p}} \mathrm{A}_{\mathrm{p}} \mathrm{k}_{\mathrm{uz}}+\mathrm{k}_{\mathrm{uz}}^{2} \mathrm{~L}_{\mathrm{e}}^{2}\right.}{4\left(3 \mathrm{E}_{\mathrm{p}} \mathrm{A}_{\mathrm{p}}+\mathrm{k}_{\mathrm{uz}} \mathrm{L}_{\mathrm{e}}^{2}+3 \mathrm{k}_{\mathrm{uzb}} \mathrm{L}_{\mathrm{e}}\right)}+\frac{\mathrm{K}_{\mathrm{uzb}}\left(3 \mathrm{E}_{\mathrm{p}} \mathrm{A}_{\mathrm{p}}+\mathrm{k}_{\mathrm{uz}} \mathrm{L}_{\mathrm{e}}^{2}\right.}{\left(3 \mathrm{E}_{\mathrm{p}} \mathrm{A}_{\mathrm{p}}+\mathrm{k}_{\mathrm{uz}} \mathrm{L}_{\mathrm{e}}^{2}+3 \mathrm{k}_{\mathrm{uzb}} \mathrm{L}_{\mathrm{e}}\right)}$ 
Where,

$\mathrm{L}_{\mathrm{e}}$ is the Length of the pile.

$\mathrm{E}_{\mathrm{p}}$ is Elastic modulus of pile.

$$
\mathrm{k}_{\mathrm{uz}}=2 \pi \mathrm{r}_{0} \frac{\mathrm{G}}{\mathrm{r}_{0} \text { in }\left(\frac{\mathrm{r}_{\mathrm{m}}}{\mathrm{r}_{\mathrm{o}}}\right)}=2 \pi \frac{\mathrm{G}}{\operatorname{In}\left(\frac{\mathrm{r}_{\mathrm{m}}}{\mathrm{r}_{\mathrm{o}}}\right)}
$$

Where,

$$
\begin{aligned}
& \mathrm{r}_{\mathrm{m}}=2.5 \mathrm{~L} \rho(1-\mathrm{v}) \\
& \mathrm{k}_{\mathrm{uzb}}=\frac{2 D G_{S}}{1-v}
\end{aligned}
$$

Where,

$\mathrm{D}$ is the diameter of the pile.

\subsubsection{Lateral Stiffness, Rotation Stiffness and Couple Stiffness}

\subsubsection{Lateral Stiffness}

$$
\mathrm{k}_{11}=\frac{60480 \mathrm{EI}^{2} \mathrm{k}_{\mathrm{uy}} \mathrm{L}+1956 \mathrm{EIk}^{2}{ }_{\mathrm{uy}} \mathrm{L}^{5}+\mathrm{k}_{\mathrm{uy}}^{3} \mathrm{~L}^{9}}{4\left(15120 \mathrm{EI}^{2}+1224 \mathrm{EIk}_{\mathrm{uy}} \mathrm{L}^{4}+\mathrm{k}_{\mathrm{uy}}^{2} \mathrm{~L}^{8}\right)}
$$

\subsubsection{Rotation Stiffness}

$$
\mathrm{k}_{55}=\frac{1512000 \mathrm{EI}^{2} \mathrm{k}_{\mathrm{uy}} \mathrm{L}^{3}+5220 \mathrm{EIk}_{\mathrm{uy}}{ }^{2} \mathrm{~L}^{7}+\mathrm{k}^{3}{ }_{\mathrm{uy}} \mathrm{L}^{11}}{300\left(15120 \mathrm{EI}^{2}+1224 \mathrm{EIk}_{\mathrm{uy}} \mathrm{L}^{4}+\mathrm{k}^{2}{ }_{\mathrm{uy}} \mathrm{L}^{8}\right)}
$$

\section{Couple Stiffness}

$k_{15}=\frac{k_{u y} L^{2}+\left(k_{u y}^{2} L^{8}+302400 E I^{2}+3060 E I k_{u y} L^{5}\right)}{40\left(15120 E I^{2}+1224 E I k_{u y} L^{4}+k_{u y}^{2} L^{8}\right)}$

Where,

$\mathrm{L}_{\mathrm{e}}$ is the Length of the pile.

$\mathrm{E}_{\mathrm{p}}$ is Elastic modulus of pile.

$$
\begin{aligned}
& \mathrm{A}_{\mathrm{p}} \text { is Area of Pile. } \\
& \mathrm{I}=\frac{1}{4} \pi r^{4} \\
& K_{u y}=\frac{0.65 E_{S}}{1-{ }^{\gamma} S^{2}}\left(\frac{E_{S} D^{4}}{E I}\right)^{\frac{1}{12}}
\end{aligned}
$$


Where,

$\mathrm{D}$ is the diameter of the pile.

Again,

$$
\begin{aligned}
& \mathrm{K}_{11}=\frac{\mathrm{k}_{14}^{2} \mathrm{k}_{33}-2 \mathrm{k}_{13} \mathrm{k}_{14} \mathrm{k}_{34}+\mathrm{k}_{13}^{2} \mathrm{k}_{44}}{\mathrm{k}_{34}^{2}-\mathrm{k}_{33} \mathrm{k}_{44}}+\mathrm{k}_{11} \\
& \mathrm{~K}_{55}=\frac{\mathrm{k}_{24}^{2} \mathrm{k}_{33}-2 \mathrm{k}_{23} \mathrm{k}_{24} \mathrm{k}_{34}+\mathrm{k}_{23}^{2} \mathrm{k}_{44}+\mathrm{k}_{22}}{\mathrm{k}_{34}^{2}-\mathrm{k}_{33} \mathrm{k}_{44}} \\
& \mathrm{~K}_{15}=\frac{\mathrm{k}_{24}^{2}\left(\mathrm{k}_{24} \mathrm{k}_{33}-\mathrm{k}_{23} \mathrm{k}_{24}\right)+\mathrm{k}_{13}\left(\mathrm{k}_{23} \mathrm{k}_{44}-\mathrm{k}_{24} \mathrm{k}_{34}\right)}{\mathrm{k}_{34}^{2}-\mathrm{k}_{33} \mathrm{k}_{44}}+\mathrm{k}_{12}
\end{aligned}
$$

Where,

$$
\begin{aligned}
& \mathrm{k}_{11}=\frac{\mathrm{EI}}{\mathrm{L}^{3}}\left(12+\frac{13}{35} \mathrm{~A}\right) \\
& \mathrm{k}_{12}=\frac{\mathrm{EI}}{\mathrm{L}^{2}}\left(6+\frac{11}{210} \mathrm{~A}\right) \\
& \mathrm{k}_{13}=\frac{\mathrm{EI}}{\mathrm{L}^{3}}\left(-12+\frac{9}{70} \mathrm{~A}\right) \\
& \mathrm{k}_{14}=\frac{\mathrm{EI}}{\mathrm{L}^{2}}\left(6-\frac{13}{420} \mathrm{~A}\right) \\
& \mathrm{k}_{22}=\frac{\mathrm{EI}}{\mathrm{L}}\left(4+\frac{1}{105} \mathrm{~A}\right) \\
& \mathrm{k}_{23}=\frac{\mathrm{EI}}{\mathrm{L}^{2}}\left(-6+\frac{13}{420} \mathrm{~A}\right) \\
& \mathrm{k}_{24}=\frac{\mathrm{EI}}{\mathrm{L}}\left(2+\frac{1}{140} \mathrm{~A}\right) \\
& \mathrm{k}_{33}=\frac{\mathrm{EI}}{\mathrm{L}^{3}}\left(12+\frac{13}{35} \mathrm{~A}\right)+\overline{\mathrm{K}}_{11} ; \\
& \mathrm{k}_{34}=\frac{\mathrm{EI}}{\mathrm{L}^{2}}\left(-6-\frac{11}{210} \mathrm{~A}\right)+\overline{\mathrm{K}}_{15} \\
& \mathrm{k}_{44}=\frac{\mathrm{EI}}{\mathrm{L}}\left(4+\frac{1}{105} \mathrm{~A}\right)+\overline{\mathrm{K}}_{55} \\
& \mathrm{And}=\frac{\mathrm{k} \mathrm{L}^{4}}{\mathrm{EI}} \\
& \mathrm{L}^{4}
\end{aligned}
$$

Where $\overline{\mathrm{K}}_{11}, \overline{\mathrm{K}}_{55}$, and $\overline{\mathrm{K}}_{15}$ are equivalent lateral, rotation and couple stiffness of lower pile segments located at the current pile segment. 


\section{CAlCULATiON}

Details of soil profile in site obtained from lab and insitu test are shown in table 1 which are used in SSI.

Table 1: Details of soil properties

\begin{tabular}{|c|c|c|c|c|c|c|c|c|}
\hline Depth $(\mathbf{m})$ & Thickness $(\mathbf{m})$ & Soil Type & & $\mathbf{N}$ & & $\mathbf{V s ( f )}$ & $\mathbf{V s ( C o r )}$ & $\boldsymbol{\rho}\left(\mathbf{g} / \mathbf{c m}^{\mathbf{3}}\right)$ \\
\hline $0-2$ & 2 & Silty clay & 18.69 & 9 & 14 & 172.143 & 261.810 & 1.35 \\
\hline $2-6$ & 4 & Silty sand & 60.045 & 31 & 35 & 215.593 & 244.915 & 1.46 \\
\hline $6-9$ & 3 & Clay & 87.615 & 29 & 27 & 202.092 & 208.883 & 1.46 \\
\hline $9-10.5$ & 1.5 & Sand & 101.4 & 71 & 65 & 252.08 & 251.205 & 1.46 \\
\hline $10.5-13.5$ & 3 & Sand & 128.97 & 50 & 40 & 223.767 & 209.978 & 1.47 \\
\hline $13.5-15$ & 1.5 & Silty sand & 142.755 & 50 & 38 & 220.911 & 202.102 & 1.47 \\
\hline
\end{tabular}

Where,

$\mathrm{Vs}(\mathrm{f})=87.8$ [Brandenberg et al. (2010)]

and,

$\mathrm{Vs}($ corr $)=\mathrm{Vs}(\mathrm{f}) *[$ Brandenberg et al. (2010)]

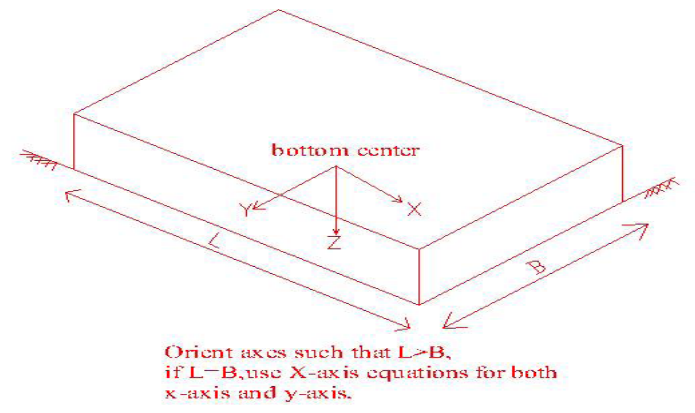

\section{Figure 44: Raft Foundation Plan}

Orient axis such that $\mathrm{L}>\mathrm{B}$, if $\mathrm{L}=\mathrm{B}$ use $\mathrm{x}$-axis equations for both $\mathrm{x}$-axis and $\mathrm{y}$-axis.

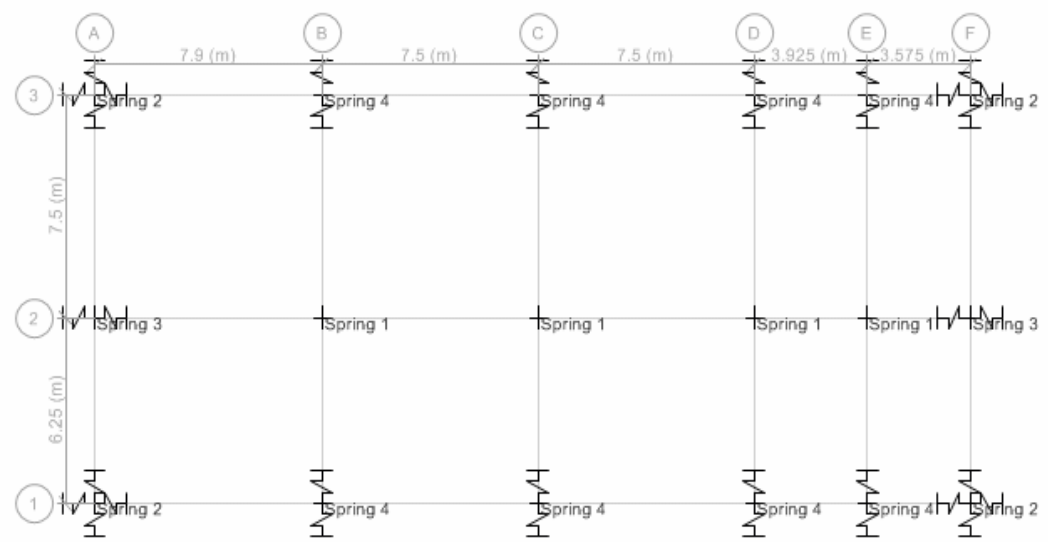

The calculated parameters of damping and springs are individual parameters acting at a defined direction. However, the assignment of the spring and dashpot occur in group.In ETABS, more than two springs cannot be assigned to a single joint. Hence, a group of individual parameter are combined to form a single joint. i.e. joint S1, S2, S3, S4, S5, S6 
and $\mathrm{S} 7$ are the set of individual spring parameters k1, k2, k3, k4, k5, k6.

Table: Spring Distribution

\begin{tabular}{|c|c|c|c|c|c|c|c|}
\hline \multirow{2}{*}{ Parameter } & \multicolumn{7}{|c|}{ Spring Type } \\
\cline { 2 - 8 } & $\mathrm{S} 1$ & $\mathrm{~S} 2$ & $\mathrm{~S} 3$ & $\mathrm{~S} 4$ & $\mathrm{~S} 5$ & $\mathrm{~S} 6$ & $\mathrm{~S} 7$ \\
\hline Spring & $\mathrm{k} 3$ & $\mathrm{k} 1, \mathrm{k} 2, \mathrm{k} 3, \mathrm{k} 4, \mathrm{k} 5$ & $\mathrm{k} 1, \mathrm{k} 3, \mathrm{k} 4$ & $\mathrm{k} 2, \mathrm{k} 3, \mathrm{k} 5$ & $\mathrm{k} 1, \mathrm{k} 4$ & $\mathrm{k} 2, \mathrm{k} 5$ & $\mathrm{k} 1, \mathrm{k} 2, \mathrm{k} 4, \mathrm{k} 5$ \\
\hline
\end{tabular}

\section{RESULT OBTAINED}
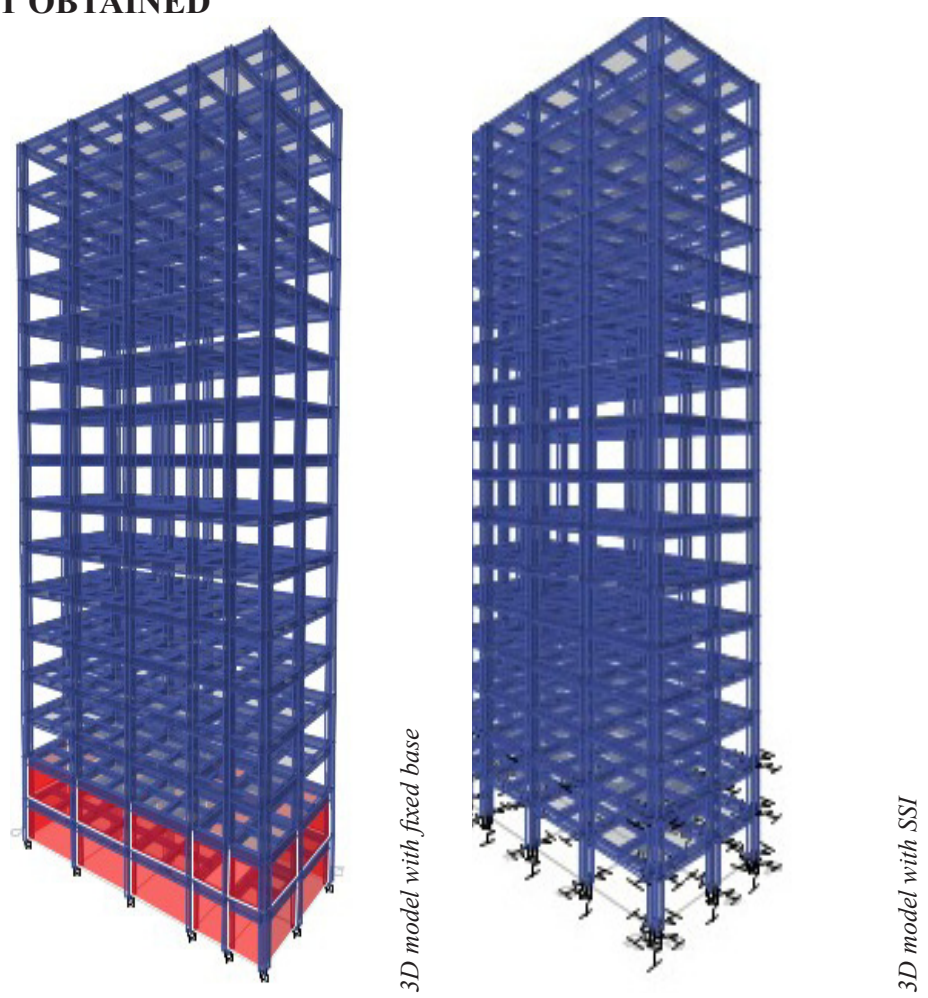

5.1 Comparison of Output-Time Period

\begin{tabular}{|l|c|r|}
\hline Mode & Fixed & SSI \\
\hline Mode 1 & 2.515 & 2.54 \\
\hline Mode 2 & 2.291 & 2.318 \\
\hline Mode 3 & 2.028 & 2.04 \\
\hline Mode 4 & 0.791 & 0.795 \\
\hline Mode 5 & 0.737 & 0.741 \\
\hline Mode 6 & 0.645 & 0.646 \\
\hline Mode 7 & 0.433 & 0.435 \\
\hline Mode 8 & 0.417 & 0.419 \\
\hline Mode 9 & 0.356 & 0.357 \\
\hline Mode 10 & 0.284 & 0.286 \\
\hline Mode 11 & 0.282 & 0.284 \\
\hline Mode 12 & 0.233 & 0.234 \\
\hline
\end{tabular}

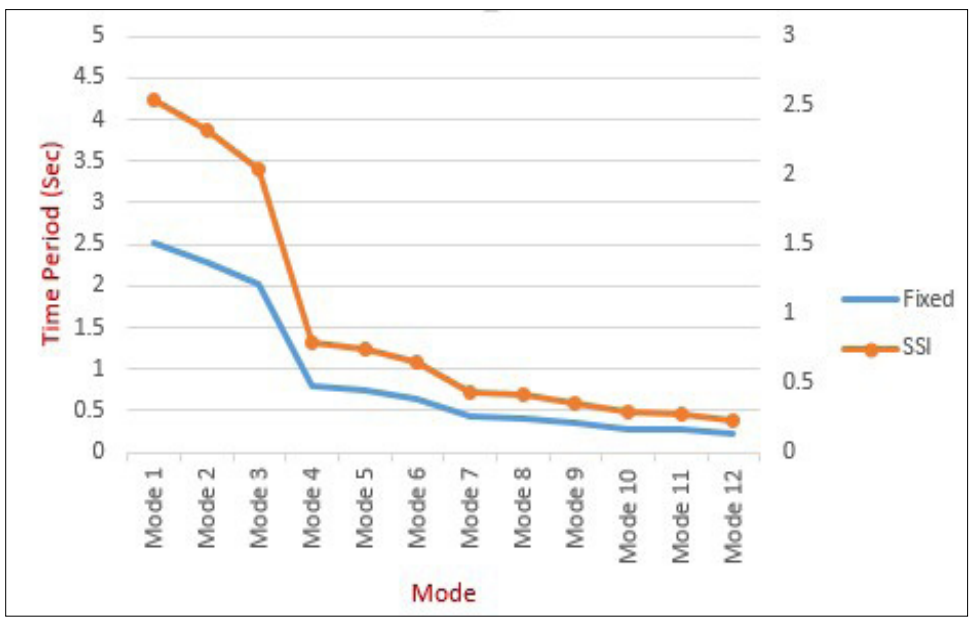


Soil-Pile-Structure Interaction ...

\subsection{Comparison of Output-Base Shear}

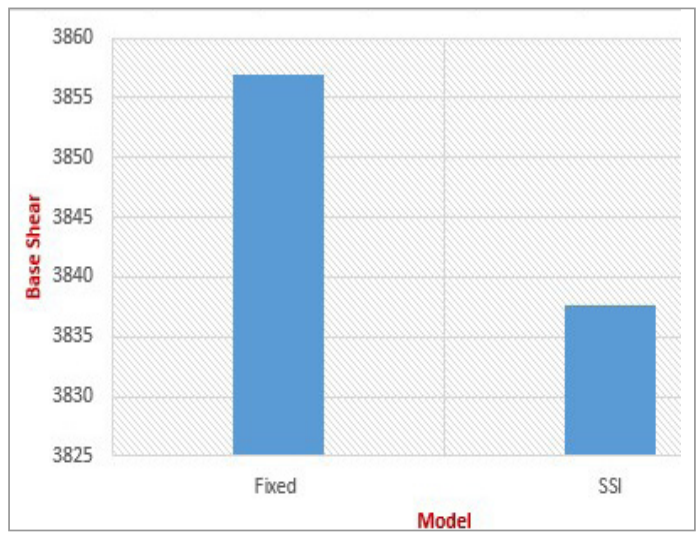

5.3 Comparison of output-Displacement w.r.t. $x$

\begin{tabular}{l|r|r|}
\hline Story & $\begin{array}{c}\text { Displacement } \\
\text { w.r.t. } x \text { for } \\
\text { fixed case } \\
\text { (mm) }\end{array}$ & $\begin{array}{c}\text { Displacement } \\
\text { w.r.t. } x \text { for } \\
\text { SSI case } \\
(\mathrm{mm})\end{array}$ \\
\hline Base & 0 & 0 \\
\hline Story1 & 0.11 & 0.172 \\
\hline Story2 & 0.318 & 0.817 \\
\hline Story3 & 4.815 & 5.075 \\
\hline Story4 & 12.074 & 12.204 \\
\hline Story5 & 20.058 & 20.031 \\
\hline Story6 & 28.464 & 28.263 \\
\hline Story7 & 36.836 & 36.502 \\
\hline Story8 & 45.032 & 44.565 \\
\hline Story9 & 52.93 & 52.337 \\
\hline Story10 & 60.41 & 59.699 \\
\hline Story11 & 67.34 & 66.527 \\
\hline Story12 & 73.582 & 72.688 \\
\hline Story13 & 78.993 & 78.041 \\
\hline Story14 & 83.428 & 82.45 \\
\hline Story15 & 86.772 & 85.802 \\
\hline Story16 & 89.066 & 88.136 \\
\hline
\end{tabular}

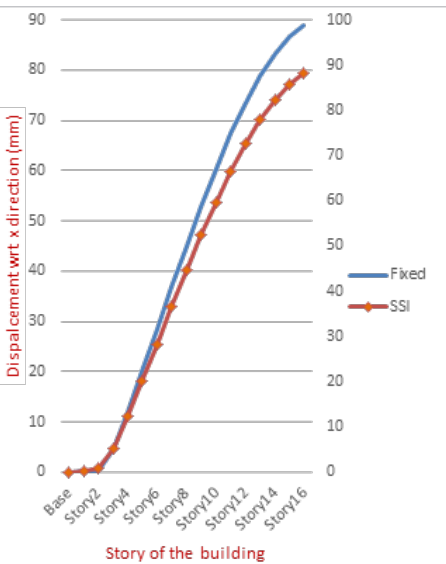

\subsection{Comparison of output-Displacement w.r.t. $y$}

\begin{tabular}{|l|r|r|}
\hline Story & $\begin{array}{c}\text { Displacement } \\
\text { w.r.t. y for } \\
\text { fixed case } \\
\text { (mm) }\end{array}$ & $\begin{array}{c}\text { Displacement } \\
\text { w.r.t. y for } \\
\text { SSI case } \\
\text { (mm) }\end{array}$ \\
\hline Base & 0 & 0 \\
\hline Story1 & 0.208 & 0.097 \\
\hline Story2 & 0.749 & 0.982 \\
\hline Story3 & 5.591 & 5.682 \\
\hline Story4 & 13.866 & 13.786 \\
\hline Story5 & 23.469 & 23.224 \\
\hline Story6 & 33.676 & 33.268 \\
\hline Story7 & 43.95 & 43.384 \\
\hline Story8 & 54.036 & 53.32 \\
\hline Story9 & 63.751 & 62.899 \\
\hline Story10 & 72.932 & 71.96 \\
\hline Story11 & 81.416 & 80.345 \\
\hline Story12 & 89.035 & 87.893 \\
\hline Story13 & 95.624 & 94.441 \\
\hline Story14 & 101.035 & 99.85 \\
\hline Story15 & 105.194 & 104.045 \\
\hline Story16 & 108.241 & 107.163 \\
\hline
\end{tabular}

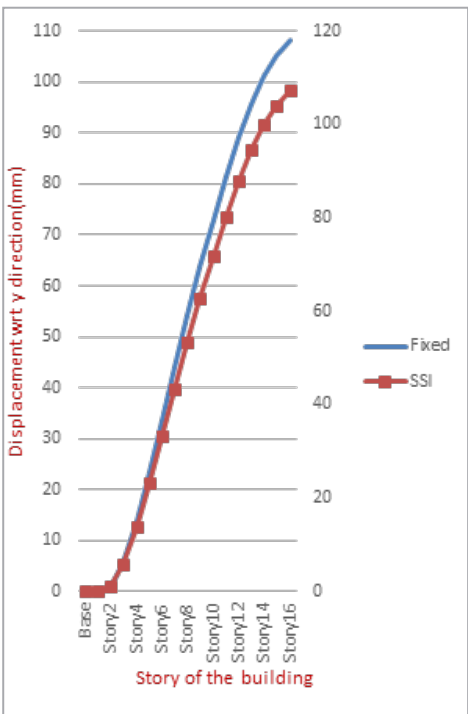




\subsection{Comparison of Output-Overturning Moment}

\begin{tabular}{|c|c|c|}
\hline Story & Fixed Base $(\mathbf{m m})$ & Winkler approach considering SSI $(\mathbf{m m})$ \\
\hline Base & 2036.112 & 1342.597 \\
\hline Story1 & 1853.323 & 1342.309 \\
\hline Story2 & 1673.221 & 1370.336 \\
\hline Story3 & 1496.792 & 1344.868 \\
\hline Story4 & 1325.277 & 1189.447 \\
\hline Story5 & 1159.104 & 1037.755 \\
\hline Story6 & 998.8839 & 890.4173 \\
\hline Story7 & 846.1835 & 750.0687 \\
\hline Story8 & 701.8881 & 618.5974 \\
\hline Story9 & 565.5118 & 495.7521 \\
\hline Story10 & 438.1567 & 383.1397 \\
\hline Story11 & 324.4241 & 286.4841 \\
\hline Story12 & 228.1264 & 208.4841 \\
\hline Story13 & 147.1475 & 141.9164 \\
\hline Story14 & 77.8231 & 79.1684 \\
\hline Story15 & 24.4523 & 26.0304 \\
\hline Story16 & 0 & 0 \\
\hline & & \\
\hline
\end{tabular}

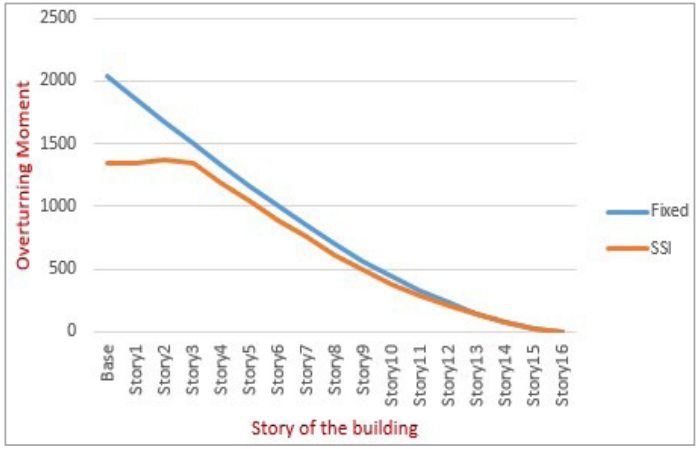

5.6 Comparison of Output-Base Shear w.r.t Time History of EI Centro Earthquake (1940)

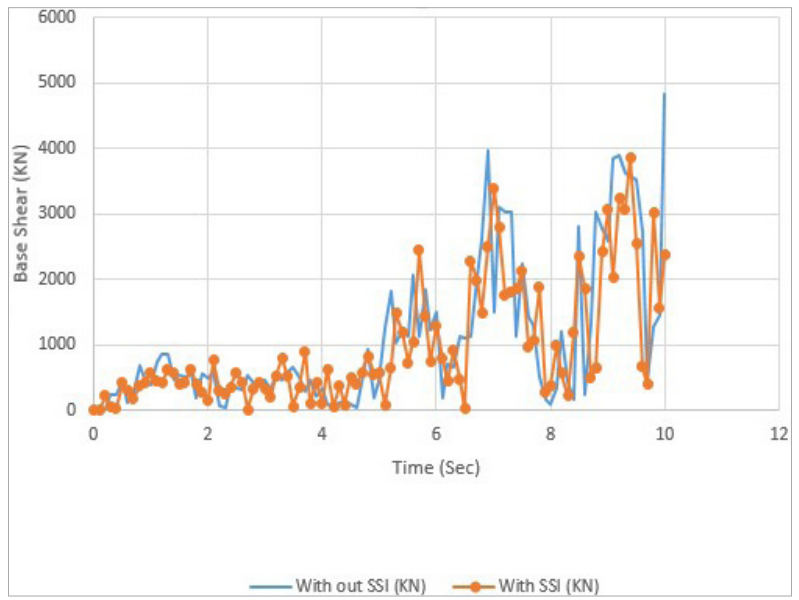

\section{CONCLUSION}

- The soil structure interaction effects increase the time period of the structure.

- It was observed that the base shear decreases in SSI based Winkler approach model than fixed based model as the base becomes more flexible by the inclusion of SSI effects.

- It was observed that the displacement decreases in SSI based Winkler approach model than fixed based model.

Overturning moment is decreased in SSI based Winkler approach model than fixed based model.

Base shear is increased in some time and decreased in rest while analyzing the model with time history of El Centro earthquake between fixed and SSI cases. $^{2}$

1. ASCE (2007), Seismic Rehabilitation of Existing Buildings, ASCE/SEI 41-06, American Society of Civil Engineers, Reston, Virginia.

2. ASCE(2010), Minimum Design Loads for Buildings and Other Structures, ASCE/SEI 7-10, 
American Society of Civil Engineers, Reston, Virginia.

3. Bielak, J.(1975), Dynamic behavior of structures with embedded foundations, Earthquake Engineering and Structural Dynamics, Vol. 3, pp. 259-274.

4. Clough, R.W., and Penzien, J.(1993), Dynamics of Structures, McGraw Hill, New York.

5. Dutta, S. and R. Roy (2002), A Critical Review on Idealization and Modeling forInteraction Among Soil Foundation-Structure System, Computers and Structures, 80, pp. 1579-1594.

6. Dutta, S., Bhattacharya, K., and R. Roy (2004), Response of Low-Rise Buildings Under Seismic Ground Excitation Incorporating Soil-Structure Interaction, Soil Dynamics and Earthquake Engineering, 24, pp. 893-914.

7. Hayashi, Y. and Takahashi, I. (2004), Soil-Structure Interaction Effects on Building Response in Recent Earthquakes, Third UJNR Workshop on Soil-Structure Interaction,Menlo Park, California, USA.

8. HienManh Nghiem (2009), Soil Pile Structure Interaction Effects on high rises under seismic shaking, University of Colorado at Denver and health science, Denver, USA.

9. IS 1893 (Part I):2002 (2002),Criteria for earthquake resistant design of structuresgeneralprovisions and buildings, Bureau of Indian standards, New Delhi,.

10. IS: 456, (2000), Plain and reinforced concrete code of practice (fourth revision), Bureau of Indian Standards, New Delhi.

11. Kramer, S. L. (1996), Geotechnical earthquake engineering, Prentice Hall, 2007, ISBN 81-3170718-0.

12. Kraus\& d. Dzakic (2013), Soil-structure interaction effects on seismic behaviour of reinforced concrete frames, josephJurajStrossmayer university of Osijek, faculty of civil engineering Osijek,Croatia.

13. Muberraeseraydemir (2009), Soil-structure interaction effects on multi-storey r/c structures, international journal of electronics; mechanical and mechatronics engineering vol.2 num.3 pp.(298-303).

14. Naim F., S. Tileyliouglu, A. Alimoradi, and J. Stewart (2008), Impact of Foundation Modeling on the Accuracy of Response History Analysis of a Tall Building,SMIP08 Seminar Proceedings.

15. Raychowdhury P. (2010), Seismic Response of Low-Rise Steel Moment-Resisting Frame (SMRF) Buildings Incorporating Nonlinear Soil-Structure Interaction (SSI), Soil Dynamics and Earthquake Engineering, 33, pp. 958-967.

16. S.Hamidrezatabaiefar, behzadfatahia and bijansamali (2012), Lateral seismic response of building frames considering dunamic soil-structure interaction effects, structural engineering and machanics, vol. 45, no. 3 311-321.

17. SushmaPulikanti (2013), Effect of Soil-Foundation-Stucture interaction on seismic behavior of pile supported frame building, International Institute of Information Technology, Hyderabad.

18. Stewart, J.P., Kim, S., Bielak, J., Dobry, R., and Power, M. (2003), Revisions to soil structure interaction procedures in NEHRP design provisions, EarthquakeSpectra, Vol. 19, No. 3, pp. 677696.

18. Tabatabaiefar R. and A. Massumi (2010),A Simplified Method to Determine Seismic Responses of Reinforced Concrete Moment Resisting Building Frames Under 20.Influence of Soil-Structure Interaction,Soil Dynamics andEarthquake Engineering, 30, pp. 1259-1267.

21.Tokimasu, K., Oh-oka, H., Satake, K., Shamoto, Y. and Asaka, Y. (1998), Effects of Lateral Ground Movements on Failure Patterns of Piles, in the 1995 Hyogoken- Nanbu Earthquake. Proc. 3rd Conf. Geotechnical Earthquake Engineering and Soil Dynamics, ASCE, Seattle, pp. 1175-1186.

22.Vaibhav Singh and Kanchan Mala (2016), Effect on Seismic Response of Building with Underground Storey Considering Soil Structure Interaction, International Journal of Engineering Research \& Technology.

23.Wolf, J.P. (1985), Dynamic Soil-Structure Interaction, Prentice-Hall, Upper Saddle River, New Jersey.

24.Wong, H.L., and Luco, J.E. (1985), Dynamic interaction between rigid foundations in a layered half space, Earthquake Engineering and Structural Dynamics, Vol. 5, No.3, pp.149-1 